\title{
Initial-boundary value problem for the spherically symmetric Einstein equations for a perfect fluid
}

\author{
Saskia Kind and Jürgen Ehlers \\ Max-Planck-Institut fur Astrophysik, Kart-Schwarzschild-Strasse 1, W-8046 Garching bei \\ München, Federal Republic of Germany
}

Received 23 August 1992

\begin{abstract}
It is shown that for a given spherically symmetric distribution of a perfect fluid on a spacelike hypersurface with boundary and a given, time-dependent boundary pressure, there exists a unique, local-in-time solution of the Einstein equations. A Schwarzschild spacetime can be atlached to the fluid body if and only if the boundary pressure vanishes. We assume a smooth equation of state for which the density and the speed of sound remain positive for vanishing pressure.
\end{abstract}

PACS numbers: 0230,0420

\section{Introduction}

An important problem in any theory of gravitation is the construction of models of isolated systems of bodies. In general relativity, models of single static, spherically symmetric fluid balls [1] and time-dependent, spherically symmetric dust solutions [2], are well understood. Until recently, nothing rigorous was known about the existence, let alone the properties of more general cases. Only in 1991 Rendall [3] solved, globally in space and locally in time, an initial value problem for a class of spacetimes containing one or several perfect fluid bodies separated by empty space, generalizing work by Makino [4] on the analogous Newtonian problem. His method, however, does not cover bodies in, or close to, static equilibrium which have a non-vanishing surface gravity, and it does not provide uniqueness of the solutions in terms of initial data.

In order to get rid of the restrictions of the Makino-Rendall work, which requires equations of state $p(\varrho)$ with $p(0)=0$ and $p^{\prime}(0)=0$, one can try to use an equation of state for which the pressure vanishes for a positive value of the mass density. Then some variables have to be discontinuous at the surfaces of the bodies, the interior and exterior evolution equations are different, and one is faced with a mixed initial-boundary value problem instead of a pure initial value problem. For quasilinear partial differential equations such as Einstein's, a problem of this type appears to be tractable at present only if, besides time, no more than one spatial coordinate is significant. This leaves one with spherically symmetric bodies in radial motion, surrounded by a Schwarzschild vacuum field.

In this paper, which is a shortened version of a part of the doctoral thesis of the first author to which the reader is referred for details [5], the mixed initial-boundary value problem for a spherically symmetric perfect fluid body is solved. We show that for a given spherically symmetric distribution of matter on a compact spacelike hypersurface and for a given boundary-value of the pressure, there exists locally in time a unique spacetime which 
can be matched to a vacuum Schwarzschild spacetime if and only if the boundary-pressure vanishes. Our results cover static and nearly static configurations which describe bodies with non-vanishing surface gravity.

This problem is solvable since the spherically symmetric system depends on two independent variables only. Courant and Lax have developed a method of solving the initial value problem for quasilinear symmetric hyperbolic systems of two independent variables which has been reviewed in $[6,7]$. Their method is generalized to mixed initial-boundary value problems in section 3.3 of this paper.

Misner and Sharp [8] have for the first time written down the spherically symmetric Einstein equations for a perfect fluid in a manageable form. Their system of equations, which is reviewed in section 2.3 below, is not symmetric hyperbolic. However, in section 3.2 it is transformed into such a system.

The neighbourhood of the bodies' centre is treated separately in 'Cartesian' coordinates because in polar-coordinates, the differential system is singular at the centre, whence the above mentioned proof fails; this is done in section 3.1.

To attach a Schwarzschild spacetime to the star it is required that the first two fundamental forms of the inner and outer sides of the star's surface agree. While the initial-boundary value problem for the fluid body alone can be solved for an arbitrarily given boundary-pressure, a Schwarzschild spacetime can be joined to it if and only if the pressure at the star's surface vanishes, as would be expected on physical grounds. The calculations concerning this junction of vacuum surroundings will not be reproduced here.

\section{Formulation of the problem}

\subsection{Choice of the equation of state}

We assume that the specific entropy of the perfect fluid be constant both in space and time, so that the equation of state has the simple form $p=p(Q)$. We require further that the density $\varrho$ and the speed of sound $s$ be positive at vanishing pressure. So for $\varrho>0$

$$
\begin{aligned}
& p(\varrho) \in C^{\infty}\left(\mathbb{R}^{+}\right) \\
& p\left(\varrho_{0}\right)=0 \quad \varrho_{0}>0 \\
& s^{2}(\varrho):=\frac{\mathrm{d}}{\mathrm{d} \varrho} p(\varrho)>0 \\
& \varrho c^{2}+p>0 .
\end{aligned}
$$

Note that $s \leqslant c$ is not assumed; it is mathematically irrelevant for the problem treated in this paper.

\subsection{Choice of coordinates}

In a globally hyperbolic, spherically symmetric spacetime occupied by a perfect fluid, there exist coordinates in which the metric has the form

$$
-c^{2} \mathrm{e}^{2 \Phi(T, R)} \mathrm{d} T^{2}+\mathrm{e}^{2 \Lambda(T, R)} \mathrm{d} R^{2}+r^{2}(T, R) \mathrm{d} \Omega^{2}
$$


where

$$
\mathrm{d} \Omega^{2}=\mathrm{d} \vartheta^{2}+\sin ^{2} \vartheta \mathrm{d} \varphi^{2}
$$

is the metric of the unit 2 -sphere, and $R, \vartheta, \varphi$ are constant along the world lines of the fluid particles (comoving, Lagrangian coordinates)

$$
u^{\mu}=\left(\mathrm{e}^{-\Phi}, 0,0,0\right) \text {. }
$$

(For a global treatment of spherically symmetric spacetimes, see [9].) The coordinates $(T, R)$ become unique if we require

$$
\Phi\left(T, R_{0}\right)=0 \quad r(0, R)=R
$$

where $R_{0}$ corresponds to the surface of the body. (The second condition excludes initial spatial configurations with a 'neck'.)

The non-vanishing components of the energy-momentum tensor $T_{\mu \nu}=(\varrho+$ $\left.\left(1 / c^{2}\right) p\right) u_{\mu} u_{1}+p g_{\mu 1}$ with respect to this coordinate system are

$$
\begin{array}{ll}
T_{r T}=\mathrm{e}^{2 \Phi} \rho c^{4} & T_{R R}=p \mathrm{e}^{2 \Lambda} \\
T_{\varphi \vartheta}=p \cdot r^{2} & T_{\varphi \varphi}=p r^{2} \sin ^{2} \vartheta .
\end{array}
$$

\subsection{Einstein and matter equations}

In a spherically symmetric spacetime, those Einstein and matter equations which contain an odd number of angle indices are satisfied identically. Due to the contracted Bianchi identities the $(\vartheta \vartheta)$ - and $(\varphi \varphi)$-components of the Einstein equations follow from the other equations and need not be considered. Only five equations for the functions $\Phi, \Lambda, r$ and $\varrho$ of $T$ and $R$ remain (we use the notation $0^{\circ}=\partial / \partial T, O^{\prime}=\partial / \partial R$ ). From $T_{; \beta}^{\alpha \beta}=0$ we obtain

$$
\begin{array}{ll}
\alpha=T: \quad & \dot{\varrho}+\left(\varrho+\frac{1}{c^{2}} p\right)\left(\dot{\Lambda}+2 \frac{\dot{r}}{r}\right)=0 \\
\alpha=R: & \left(\varrho c^{2}+p\right) \Phi^{\prime}+p^{\prime}=0 .
\end{array}
$$

and from $G_{\mu \nu}=\frac{8 \pi G}{c^{4}} T_{\mu \nu}$

$$
\begin{array}{rlrl}
(\mu \nu)=(T T): & \frac{8 \pi G}{c^{2}} \varrho & =\frac{1}{r^{2}}\left[1-\left(r^{\prime}\right)^{2} \mathrm{e}^{-2 \Lambda}+\frac{1}{c^{2}}(\dot{r})^{2} \mathrm{e}^{-2 \Phi}\right] \\
& +\frac{1}{c^{2}} \frac{2}{r} \dot{r} \dot{\Lambda} \mathrm{e}^{-2 \Phi}-\frac{2}{r}\left(r^{\prime \prime}-r^{\prime} \Lambda^{\prime}\right) \mathrm{e}^{-2 \Lambda} \\
(\mu \nu)=(R R): \quad & \frac{8 \pi G}{c^{4}} p=-\frac{1}{r^{2}}\left[1-\left(r^{\prime}\right)^{2} \mathrm{e}^{-2 \Lambda}+\frac{1}{c^{2}}(\dot{r})^{2} \mathrm{e}^{-2 \Phi}\right] \\
& +\frac{2}{r} r^{\prime} \Phi^{\prime} \mathrm{e}^{-2 \Lambda}-\frac{1}{c^{2}} \frac{2}{r}(\ddot{r}-\dot{r} \dot{\Phi}) \mathrm{e}^{-2 \Phi} \\
(\mu \nu)=(R T): \quad & \dot{r} \Phi^{\prime}+r^{\prime} \dot{\Lambda}-\dot{r}^{\prime}=0 .
\end{array}
$$


Equation (2.7) can be integrated at once, taking into account (2.4)

$$
\Phi=-\int_{Q 0}^{\varrho} \frac{s^{2}(\bar{Q})}{\bar{Q} c^{2}+p(\bar{Q})} \mathrm{d} \bar{Q} .
$$

$T$ then measures proper time at the star's surface, and $\Phi$ is henceforth to be considered as a function of $Q$.

It is useful to define a radial velocity

$$
v:=\mathrm{e}^{-\Phi} \dot{r}
$$

and a mean density of the matter within a ball of coordinate radius $R$

$$
\mu:=\frac{3}{r^{3}} \int_{0}^{R} \bar{e} \bar{r}^{2} \bar{r}^{\prime} \mathrm{d} \bar{R}
$$

With these variables, $(2.10)$ can be written as

$$
\dot{\Lambda}=\mathrm{e}^{\Phi} \frac{\mathrm{v}^{\prime}}{r^{\prime}}
$$

and the local energy balance (2.6) takes the form

$$
\dot{Q}=-\left(Q+\frac{p}{c^{2}}\right)\left(\frac{v^{\prime}}{r^{\prime}}+2 \frac{v}{r}\right) \mathrm{e}^{\Phi} .
$$

Moreover, $(2.8)$ and the regularity of the metric at the centre imply

$$
r^{2} \mathrm{e}^{-2 \Lambda}=1+\frac{v^{2}}{c^{2}}-\frac{8 \pi G}{3 c^{2}} \mu r^{2}
$$

and (2.9) can be recognized as the equation of motion

$$
\mathrm{e}^{-\Phi} \dot{v}=-\frac{r^{\prime} \mathrm{e}^{-2 \Lambda}}{Q+\left(p / c^{2}\right)} p^{\prime}-4 \pi G r\left(\frac{1}{3} \mu+\frac{p}{c^{2}}\right)
$$

which generalizes the Tolman-Oppenheimer-Volkoff equation of hydrostatic equilibrium. These equations further imply

$$
\mathrm{e}^{-\Phi} \dot{\mu}=-3 \frac{v}{r}\left(\mu+\frac{p}{c^{2}}\right)
$$

The last equation is equivalent to the adiabatic energy balance $\mathrm{d} E=-p \mathrm{~d} V$ for the 'total energy' $E:=\frac{4}{3} \pi \mu r^{3} c^{2}$ within the $R$-ball. Thus, we have obtained a system of evolution equations for the variables $r, v, \varrho, \Lambda, \mu$. Moreover, these variables are subject to the constraints (2.13) and (2.16). 


\subsection{Initial and boundary data}

On both physical and mathematical grounds we expect that $\mathrm{Q}(0, R)=: \hat{Q}(R)$ and $v(0, R)=$ : $\hat{v}(R)$ can be chosen as free initial data. Obviously, $\hat{v}(0)=0$. In contrast to the Newtonian case, $\hat{Q}$ and $\hat{v}$ are constrained by the inequality

$$
\frac{8 \pi G}{R c^{2}} \int_{0}^{R} \hat{\varrho}(\vec{R}) \bar{R}^{2} \mathrm{~d} \bar{R}<1+\frac{(\hat{v}(R))^{2}}{c^{2}}
$$

as follows from (2.4), and (2.13) and (2.16). This inequality bounds the 'compactness' of the initial mass distribution, similar to the Schwarzschild-Buchdahl inequality [10], $2 G m / R c^{2}<\frac{8}{9}$ for static bodies. Misner and Hemandez [11] have pointed out that if the left-hand side of (2.19) exceeds unity, gravitational collapse to a singularity is inevitable. It should be noted that, since $\hat{v}$ is the proper time derivative of the 'areal radius' of a fluid particle, not the velocity in any local inertial system, it is not constrained by $\hat{v}<c$.

The initial data for $r, \mu$ and $\Lambda$ follow from (2.4), (2.13) and (2.16), respectively. It is easily checked that the specified initial data determine the metric $\hat{g}_{a b}$ of the initial hypersurface $T=0,0 \leqslant R \leqslant R_{0}$, as well as its extrinsic curvature (in the spacetime to be constructed), $\hat{K}_{a b}$. In fact

$$
\begin{aligned}
& \hat{g}_{a b} \mathrm{~d} x^{a} \mathrm{~d} x^{b}=\mathrm{e}^{2 \hat{\Lambda}} \mathrm{d} R^{2}+R^{2} \mathrm{~d} \Omega^{2} \\
& \hat{K}_{a b} \mathrm{~d} x^{a} \mathrm{~d} x^{b}=\mathrm{e}^{2 \hat{\Lambda}} \frac{\hat{v}^{\prime}}{c} \mathrm{~d} R^{2}+\frac{\hat{v}}{c} R \mathrm{~d} \Omega^{2} .
\end{aligned}
$$

At the surface of the fiuid body, formed by those particles which form a sphere of radius $R_{0}$ at the initial time, the pressure can be specified freely. The required boundary value is given by the function $p\left(T, R_{0}\right)=: \tilde{p}(T)$, subject to the constraint $\tilde{p}(0)=p\left(\hat{\varrho}\left(R_{0}\right)\right)$.

The problem to be solved in the next section consists of proving existence and uniqueness of suitably differentiable (see below) solutions to the evolution equations (2.12), (2.17), (2.15), (2.14), (2.18) for the variables $r, v, \varrho, \Lambda, \mu$, subject to the specified initial and boundary data. The proof will show that the constraints (2.13), (2.16) are satisfied as a consequence of the evolution equations since the data have been chosen to obey the constraints initially. Thus, a solution of the Einstein equations is indeed obtained. Note that all preceding considerations hold if $r, v, \varrho, \Lambda, \mu$ are three times continuously differentiable.

Remark. If one substitutes $\Phi \rightarrow \Phi / c^{2}$ in the foregoing equations and then puts $1 / c=$ 0 , one obtains the equations of the corresponding Newtonian problem in Lagrangian formulation; $\Phi$ then denotes the Newtonian potential.

\section{Existence and uniqueness of the solution}

We show local existence and uniqueness of the solution, first in a domain $G_{1}$ surrounding the centre of the body, and then in a neighbourhood $G_{2}$ of its boundary, as indicated in figure 1. In the overlap $G_{1} \cap G_{2}$, the two solutions agree due to uniqueness. 


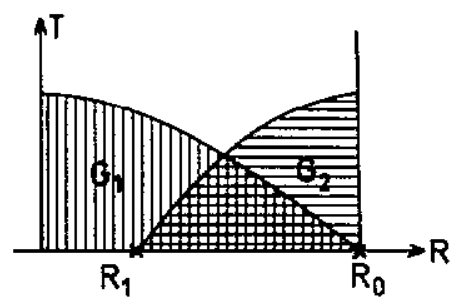

Figure 1. The two solution domains $G_{1}, G_{2}$ and their intersection.

\subsection{Solution in the domain $G_{\mathrm{t}}$}

Rendall has shown [3] that the Einstein and matter equations for a perfect fluid can, by use of harmonic coordinates and an appropriate density variable, be brought to such a form that they are symmetric hyperbolic in a neighbourhood of appropriately chosen initial data. Such initial data are obtained from our data as follows. We introduce 'Cartesian' coordinates in the initial hypersurface, $x:=R \sin \vartheta \cos \varphi, y:=R \sin \vartheta \sin \varphi, z:=R \cos \vartheta$. We compute the metric $\hat{g}_{a b}$ and the extrinsic curvature $\hat{K}_{a b}$ in these coordinates, using (2.20), and then put $\hat{\dot{g}}_{a b}=2 \hat{K}_{a b}$ (where the dot now refers to the harmonic time-coordinate in the spacetime to be constructed). Moreover, we put $\hat{g}_{00}=-c^{2}, \hat{g}_{0 b}=0$ for $a, b \in\{x, y, z\}$. The initial data for $\dot{g}_{00}$ and $\dot{g}_{0 b}$ are provided by the harmonicity condition, and the data for the matter variables are $\hat{\varrho}$ and $\hat{u}^{a}=0$. According to [3], for given $C^{\infty}$ data there exists locally in time a unique $C^{\infty}$ solution of the Einstein and matter equations.

The requirement that $\hat{g}_{a b}, \hat{K}_{a b}$ and $\hat{Q}$ be $C^{\infty}$ with respect to Cartesian coordinates restricts our two basic initial data $\hat{\varrho}, \hat{v}$ as follows: for $R \in\left[0, R_{0}\right], \hat{\varrho}(R)=f(R)$ and $\hat{v}(R)=R h(R)$, where $f$ and $h$ are even, smooth $\left(C^{\infty}\right)$, real-valued functions on $\mathbb{R}$, and $f \geqslant 0$. Therefore, $\hat{v}(0)=0$, and at $R=0$, all odd-order (right-)derivatives of $\hat{\varrho}$ and $\hat{v} / R$ vanish. Spherical symmetry of the solution corresponding to such data follows via uniqueness from the spherical symmetry of the data.

\subsection{Symmetric hyperbolic equations}

To prove existence and uniqueness in the domain $G_{2}$, we first cast the equations of the preceding section into the form of a quasilinear, symmetric hyperbolic system of first order with constraints.

Instead of $r$ and $\varrho$ we use logarithmic variables

$$
\begin{aligned}
Q & :=\ln (R / r) \\
\mathcal{L} & :=\int_{Q 0}^{Q} \frac{s(\bar{\varrho})}{\bar{Q}+\left(1 / c^{2}\right) p(\bar{\varrho})} \mathrm{d} \bar{Q} .
\end{aligned}
$$

Since the relation between $Q$ and $\mathcal{L}$ is invertible, $Q, p, s$ and $\Phi$ can be considered as known functions of $\mathcal{L}$, given an equation of state.

To obtain quasilinear equations, we introduce the variable

$$
\omega:=r^{\prime}
$$

$\dot{\omega}$ can easily be computed by (2.12). Thus we get a first-order, quasilinear, hyperbolic system for $Q, v, \mathcal{L}, \Lambda, \mu, \omega$. To achieve symmetry as well, we use

$$
w:=v / r
$$


instead of $v$ and introduce two additional variables

$$
\begin{aligned}
& X:=\mathrm{e}^{-\Lambda} \mathcal{L}^{\prime} \\
& Y:=\frac{v^{\prime}}{\omega}+2 \frac{v}{r} .
\end{aligned}
$$

A system of equations for $Q, \mathcal{L}, \Lambda, w, \omega, X, Y$ and $\mu$ is now obtained in the following way: (2.15), (2.17), (2.14), (2.12) and (2.18) provide evolution equations for $\mathcal{L}, w, \Lambda, \omega$, and $\mu$, respectively. Evolution equations for $X$ and $Y$ are obtained by differentiating the respective definitions with respect to time and eliminating the time derivatives on the right-hand sides. Equations (2.12) and (3.4) give an evolution equation for $Q$. The equations (3.3), (3.5), (3.6) and the derivatives with respect to $R$ of (2.13) and (2.16) are constraints.

Thus, we have obtained the following basic system of equations

$$
\begin{aligned}
& \dot{X}+s \mathrm{e}^{\Phi-\Lambda} Y^{\prime}=\mathrm{e}^{\Phi}\left[X Y\left(\frac{s^{2}}{c^{2}}-\frac{\mathrm{d} s}{\mathrm{~d} \mathcal{L}}-1\right)+2 w X\right] \\
& \dot{Y}+s \mathrm{e}^{\Phi-\lambda} X^{\prime}=\mathrm{e}^{\phi}\left[X^{2}\left(\frac{s^{2}}{c^{2}}-\frac{\mathrm{d} s}{\mathrm{~d} \mathcal{L}}\right)-2 s \omega \mathrm{e}^{\ell-\Lambda} \frac{X}{R}\right. \\
& \left.-(Y-2 w)^{2}-2 w^{2}-4 \pi G\left(\varrho+\frac{3 p}{c^{2}}\right)\right] \\
& \dot{Q}=-\mathrm{e}^{\Phi} w \\
& \dot{\Lambda}=\mathrm{e}^{\phi}(Y-2 w) \\
& \dot{\mathcal{L}}=-\mathrm{e}^{\Phi} s Y \\
& \dot{w}=-\mathrm{e}^{\Phi}\left[s \omega \mathrm{e}^{Q-\Lambda} \frac{X}{R}+w^{2}+\frac{4 \pi G}{3}\left(\mu+\frac{3}{c^{2}} p\right)\right] \\
& \dot{\omega}=\mathrm{e}^{\Phi}\left[\omega(Y-2 w)-\frac{s}{c^{2}} \mathrm{e}^{\Lambda-Q} R X w\right] \\
& \dot{\mu}=-3 \mathrm{e}^{\Phi} w\left(\mu+\frac{1}{c^{2}} p\right) \\
& Z_{1}:=R Q^{\prime}+\omega^{Q}-1=0 \\
& Z_{2}:=\mathcal{L}^{\prime}-\mathrm{e}^{\wedge} X=0 \\
& Z_{3}:=\operatorname{Re}^{-Q} w^{\prime}-\omega(Y-3 w)=0 \\
& \mathrm{Z}_{4}:=\frac{4 \pi G}{3}\left[\operatorname{Re}^{-Q} \mu^{\prime}+3 \omega(\mu-\varrho)\right]=0 \\
& Z_{5}:=\mathrm{e}^{-2 \Lambda}\left(\omega^{\prime}-\omega \Lambda^{\prime}\right)+\frac{1}{c^{2}} R \mathrm{e}^{-Q}\left[\frac{4 \pi G}{3}(3 \varrho-\mu)-w(Y-2 w)\right]=0 .
\end{aligned}
$$


The characteristics of the system (3.7) are the integral curves of

$$
\dot{R}_{1,2}= \pm s \mathrm{e}^{\Phi-\Lambda}
$$

which correspond to outgoing and incoming sound waves, and the fluid streamlines, $R=$ constant. Gravitational, i.e. null characteristics, do not occur since spherically symmetric gravitational waves do not exist.

Note that at two places in equations (2.7), $R$ occurs in the denominator, in the form $X / R$. The resulting singular behaviour of the equations at $R=0$, which is due to the use of polar coordinates, forced us to treat the centre separately, as remarked above.

A straightforward but lengthy calculation shows that, for a $C^{1}$ solution $X, \ldots, \mu$ of (3.7), the quantities $Z_{1}, \ldots, Z_{5}$ defined by (3.8) obey a linear, homogeneous system of the form

$$
\dot{Z}_{k}=\sum_{\ell=1}^{5} A_{k \ell} Z_{\ell}
$$

where the $A_{k \ell}$ are continuous functions of $X, \ldots, \mu$. Hence the constraints $Z_{k}=0$ are satisfied for all time if they are satisfied at $T=0$.

From the initial data $\hat{Q}(R), \hat{v}(R)$ specified in section 3.1 above we immediately obtain, taking into account (2.4)

$$
\mathcal{L}(0, R):=\mathcal{L}(\hat{\varrho}(R)) \quad w(0, R)=\frac{\hat{v}(R)}{R} \quad Q(0, R)=0 .
$$

The equations (2.13), (2.16), (3.3), (3.5) and (3.6) then provide the initial data for $\mu, \Lambda, \omega$, $X$ and $Y$, which obey the constraints $Z_{k}(0, R)=0$. These data are smooth on $\left[R_{1}, R_{0}\right]$, where $0<R_{1}<R_{0}$.

From the smooth boundary value $\tilde{p}(T)$ we obtain $\mathcal{L}\left(T, R_{0}\right)$, and the fifth equation (3.7) then provides

$$
\tilde{Y}(T)=Y\left(T, R_{0}\right)=-\left[\mathrm{e}^{-\Phi(\mathcal{L})} \frac{\dot{\mathcal{L}}}{s(\mathcal{L})}\right]\left(T, R_{0}\right)
$$

In the following paragraph the main theorem is proved which can be applied to our problem if we require $\hat{\varrho}, \hat{v}$ and $\tilde{p}$ to be chosen so that the comer conditions of that theorem are satisfied.

Remark. The parameter $\lambda=1 / c^{2}$ enters $(2.7),(2.8)$ only as a factor of undifferentiated terms (relativistic 'corrections' to the corresponding Newtonian equations). Therefore, the solutions depend continuously and differentiably on $\lambda$, even at $\lambda=0$, as can be verified by a slight extension of the proof given below. An analogous limit statement does not follow from the argument in section 3.1 above, since the reduced Einstein equation ceases to be hyperbolic at $\lambda=0$. Although we also expect a Newtonian limit to exist in that case, we have not established it.

\subsection{Existence and uniqueness theorem}

Existence and uniqueness of a $C^{k}$ solution in a domain $G_{2}$ is proved by applying to the system (3.7) the following theorem: 
Theorem. Let the system

$$
\begin{aligned}
& \dot{X}+a(U) Y^{\prime}=F(X, Y, U, R) \\
& \dot{Y}+a(U) X^{\prime}=G(X, Y, U, R) \\
& \left.\dot{U}_{j}=H_{j}(X, Y, U, R)\right) \quad j=1, \ldots, p
\end{aligned}
$$

be given, where $F, G, H_{j}$ and $a$ are $C^{k+1}$ functions of their arguments (for $R \geqslant 0$ ), and $a$ is always positive. Further, let $C^{k+1}$ initial values $\hat{X}, \hat{Y}$ and $\hat{U}$ on $\left[R_{1}, R_{0}\right]$, and the $C^{k+1}$ boundary value $\tilde{Y}(T)$ be given. The data are assumed to satisfy the corner conditions according to which $\tilde{Y}(0), \dot{\tilde{Y}}(0), \ldots, \stackrel{k+1}{\tilde{Y}}(0)$ equal the values of $Y, \dot{Y}, \ldots, \stackrel{K}{Y}^{k+1}$ at $\left(0, R_{0}\right)$ which are determined by (3.9) and the initial data. Then (3.9) has a unique $C^{k}$ solution on a compact trapezoidal domain $\mathcal{T}$ as indicated in figure 2, provided $T_{0}$ and the slope of the left boundary of $\mathcal{T}$ are sufficiently small.

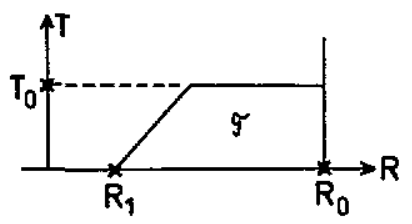

Figure 2. The trapezoidal domain $T$.

Proof. Let

$$
A:=X+Y \quad B:=X-Y
$$

and use the abbreviations

$$
U:=\left(u_{1}, \ldots, u_{p}\right) \quad E:=(A, B, U) .
$$

Then the system (3.9) can, in an obvious notation, be rewritten as

$$
\begin{aligned}
& \left(\frac{\partial}{\partial T}+a \frac{\partial}{\partial R}\right) A=L(E, R) \\
& \left(\frac{\partial}{\partial T}-a \frac{\partial}{\partial R}\right) B=M(E, R) \\
& \frac{\partial}{\partial T} U=N(E, R)
\end{aligned}
$$

where $L, M$ and $N$ are $C^{k+1}$ functions of their arguments. This system of evolution equations is in canonical form, i.e. each unknown is differentiated in a characteristic direction only.

As before, we have $C^{k+1}$ initial data $\hat{E}$ for all components of $E$. $\tilde{Y}$ provides the boundary value for $A-B$

$$
(A-B)\left(T, R_{0}\right)=2 \tilde{Y}(T) .
$$


In order to transform (3.11) into a system of integral equations, we integrate along the characteristics. We denote by $R_{1,2}(T ; \bar{T}, \bar{R})$ the sound characteristics which pass through the point $(\bar{T}, \breve{R})$

$$
\frac{\mathrm{d}}{\mathrm{d} T} R_{1,2}= \pm a\left[U\left(T, R_{1,2}\right)\right]
$$

By integrating the first of equations (3.11) we obtain

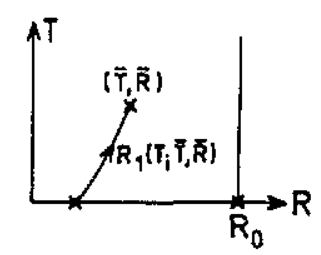

$A(\bar{T}, \bar{R})=\hat{A}\left(R_{1}(0 ; \bar{T}, \bar{R})\right)+\left.\int_{0}^{\bar{T}} \mathrm{~d} T L(E, R)\right|_{R=R_{1}(T ; \bar{T}, \bar{R}\rangle}$

In the case of $B$ we have to distinguish two cases:

(1) $R_{2}(T ; \bar{T}, \bar{R})$ starts from $T=0$

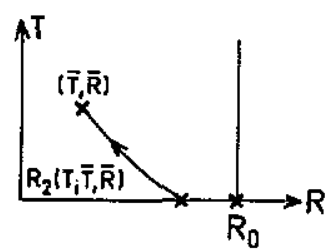

$B(\bar{T}, \bar{R})=\hat{B}\left(R_{2}(0 ; \bar{T}, \bar{R})+\left.\int_{0}^{\bar{T}} \mathrm{~d} T M(E, R)\right|_{R=R_{2}(T ; \bar{T}, \bar{R})}\right.$

(2) $R_{2}(T ; \bar{T}, \bar{R})$ starts from $R=R_{0}$. In this case we define $T_{R}(\bar{T}, \bar{R})$ implicitly by

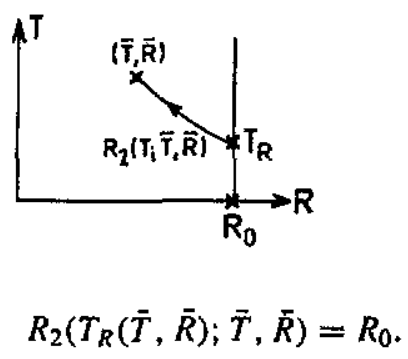


We then have to express $B\left(T_{R}, R_{0}\right)$ in terms of known quantities.

For $B$, we use $(3.3) ; B\left(T_{R}, R_{0}\right)=A\left(T_{R}, R_{0}\right)-2 \tilde{Y}\left(T_{R}\right)$

$$
\begin{gathered}
B(\bar{T}, \bar{R})=\hat{A}\left(R_{\mathrm{t}}\left(0 ; T_{R}, R_{0}\right)\right)-2 \tilde{Y}\left(T_{R}\right)-\left.\int_{0}^{T_{R}} \mathrm{~d} t L(E, R)\right|_{R=R_{1}\left(T ; T_{R}, R_{0}\right)} \\
+\left.\int_{T_{R}}^{\bar{T}} \mathrm{~d} t M(E, R)\right|_{R=R_{2}(T ; \bar{T}, \tilde{R}) \cdot}
\end{gathered}
$$

The equation for $U$ is the simplest one

$$
U(\bar{T}, \bar{R})=\hat{U}(\bar{R})+\int_{0}^{\bar{T}} \mathrm{~d} T N(E, \bar{R}) .
$$

We now construct a sequence $\left(E^{n}\right), n=0,1,2, \ldots$ of approximate solutions on a domain $\mathcal{T}$ as indicated in the theorem. We choose a value $R_{1}$ between 0 and $R_{0}$ and an $a$ priori bound $T_{\max }$ for the maximum time up to which the $E^{n}$ are to be determined. The functions $E^{n}$ will be constructed such that they and their derivatives take on the given initial and boundary data on $R_{1} \leqslant R \leqslant R_{0}$ and $0 \leqslant T \leqslant T_{\max }$, respectively. Moreover, we prescribe a priori ranges for the functions $E_{j}^{n}$ and their derivatives on the domain $\left\{(T, R) \mid 0 \leqslant T \leqslant T_{\max }, R_{1} \leqslant R \leqslant R_{0}\right\}$, compatible with the data, according to the inequalities

$$
\left|E_{j}^{n}\right| \leqslant S_{0} \quad\left|D^{l} E_{j}^{n}\right| \leqslant S_{l} \leqslant S \quad(l \leqslant k+1)
$$

where $D^{l}$ denotes differentiations of order $l$; the constants $S_{i}$ will be chosen later. Let $\lambda_{i, 2}$ denote the minimum, respectively maximum, of $a(U)$ on the domain $\left|u_{j}\right| \leqslant S_{0} ; 0<\lambda_{1}<$ $\lambda_{2}<\infty$. Then the slopes $\mathrm{d} T / \mathrm{d} R$ of the sound characteristics in the domain considered will be in the range $1 / \lambda_{2} \leqslant \mathrm{~d} T / \mathrm{d} R \leqslant 1 / \lambda_{1}$. We therefore now define the left boundary of $\mathcal{T}$ to be the line through $\left(0, R_{1}\right)$ with slope $1 / \lambda_{2}$.

The sequences $E^{n}$ and $R_{1,2}^{n}$ are defined on $\mathcal{T}$ as follows. $E^{0}$ is chosen freely, apart from the restrictions imposed by the data and the inequalities (3.18). The initial characteristics $R_{\mathrm{1}, 2}^{0}$ are defined by

$$
R_{1,2}^{0}(T ; \bar{T}, \bar{R})=\bar{R} \pm \frac{T-\bar{T}}{\lambda_{1}}
$$

$E^{n+1}$ is computed by means of (3.14), (3.15) and (3.17) where, of course, $E^{n}$ and $R_{1,2}^{n}$ are to be used on the right-hand sides. $R_{1,2}^{n+1}$ are found as the solutions of

$$
\frac{\mathrm{d}}{\mathrm{d} T} R_{1,2}^{n+1}(T ; \bar{T}, \bar{R})= \pm a\left[U^{n+1}\left(T, R_{1,2}^{n+1}(T ; \bar{T}, \bar{R})\right)\right] \quad R_{1,2}^{n+1}(\bar{T} ; \bar{T}, \bar{R})=\bar{R} .
$$

In accordance with (3.19a), we impose the $a$ priori bounds

$$
\begin{aligned}
& \left|\frac{\partial}{\partial \bar{R}} R_{1,2}^{n}(T ; \bar{T}, \bar{R})\right| \leqslant 2 \quad\left|\frac{\partial}{\partial \bar{T}} R_{1,2}^{n}(T ; \bar{T}, \tilde{R})\right| \leqslant \lambda_{1}^{-1}+\lambda_{2} \\
& \left|D^{l} R_{1,2}^{n}\right| \leqslant \sigma_{l} \quad(2 \leqslant l \leqslant k+1) .
\end{aligned}
$$


In the original method of Courant and Lax, the approximate characteristics are treated on the same footing as the approximate solutions, i.e. they are obtained by iterating an integral equation. That is not possible in the case of a mixed initial-boundary value problem because each $R_{2}^{n}(T ; \bar{T}, \bar{R})$ is defined on a different $T$ interval. Note also that we treat the quasilinear system directly, without using results for semilinear systems.

In order to check whether the inequalities (3.18), (3.20) are preserved during the iteration, we estimate the right-hand sides of the iteration equations and the required derivatives of them with respect to $\bar{R}$ and $\bar{T}$. The constants $S_{i}, \sigma_{i}$ are then chosen so that, for a sufficiently small, positive $T_{0}\left(\leqslant T_{\max }\right),(3.18)$ and (3.20) remain valid. For such a choice to be possible it is crucial that the bounds of $L, M$ and $N$ and their derivatives on the domain defined by (3.18) and $R_{1}$ depend on $S_{0}$ only.

$R_{2}^{n}\left(T ; 0, R_{0}\right)$ divides $T$ into two subdomains $\mathcal{T}_{1}^{n}$ and $T_{2}^{n}$ in which $B^{n+1}$ is defined differently, corresponding to $(3.15 a, b)$, respectively. The comer conditions of the theorem guarantee that on the boundary between $\mathcal{T}_{1}^{n}$ and $\mathcal{T}_{2}^{n}$, both definitions of $B^{n+1}$ and $B^{n+l^{\prime}}$ and their derivatives agree.

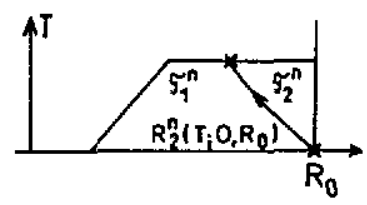

In order to prove convergence of the sequence of approximate solutions we form the differences $E^{n+1}-E^{n}$ and estimate the right-hand sides of the equations with the help of the mean value theorem. When estimating $B^{n+1}-B^{n}$ we have to distinguish three cases:
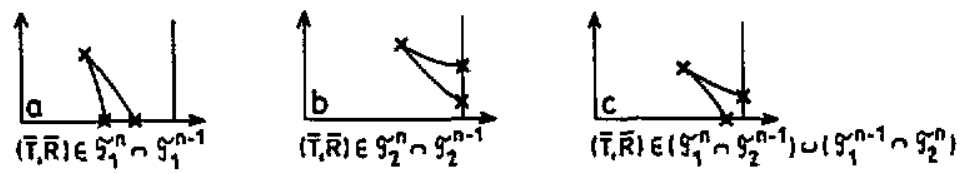

The first case does not cause any difficulties. In the other two cases, attention has to be paid to the different domains of definition of $R_{2}^{n}$ and $R_{2}^{n-1}$, respectively. In the second case, let

$$
T_{R}^{m} \geqslant T_{R}^{\bar{m}}
$$

where $(m, \bar{m})=(n, n-1)$ or $(n-1, n)$.

Then we obtain:

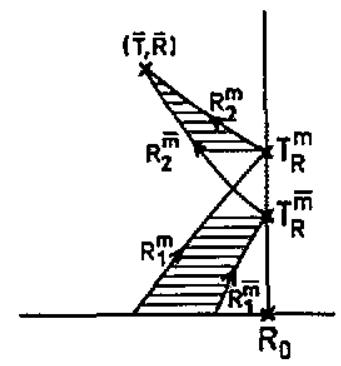




$$
\begin{aligned}
\pm\left(B^{n+1}-B^{n}\right) & (\bar{T}, \bar{R}) \\
= & -2\left[\tilde{Y}\left(T_{R}^{m}\right)-\tilde{Y}\left(T_{R}^{\bar{m}}\right)\right]+\left[\hat{A}\left(R_{1}^{m}\left(0 ; T_{R}^{m}, R_{0}\right)\right)-\hat{A}\left(R_{1}^{\bar{m}}\left(0 ; T_{R}^{\bar{m}}, R_{0}\right)\right)\right] \\
& -\int_{0}^{T_{R}^{\bar{m}}} \mathrm{~d} T\left[\left.L\left(E^{m}, R\right)\right|_{R=R_{1}^{m}\left(T: T_{R}^{m}, R_{n}\right)}-\left.L\left(E^{\bar{m}}, R\right)\right|_{R=R_{1}^{m}\left(T ; T_{R}^{m}, R_{0}\right)}\right] \\
& +\int_{T_{R}^{m}}^{\tilde{T}} \mathrm{~d} T\left[\left.M\left(E^{m}, R\right)\right|_{R=R_{2}^{m}(T: \bar{T}, \bar{R})}-\left.M\left(E^{\bar{m}}, R\right)\right|_{\left.R=R_{2}^{m}(T ; \bar{T}, \bar{R})\right]}\right. \\
& -\int_{T_{R}^{m}}^{T_{R}^{m}} \mathrm{~d} T\left[\left.L\left(E^{m}, R\right)\right|_{R=R_{1}^{m}\left(T: T_{R}^{m}, R_{0}\right)}+\left.M\left(E^{\bar{m}}, R\right)\right|_{\left.R=R_{2}^{m}(T ; \bar{T}, \bar{R})\right] .}\right.
\end{aligned}
$$

When estimating the right-hand sides, expressions of the form constant $\times\left|T_{R}^{m}-T_{R}^{\bar{m}}\right|$ will be obtained, which can be estimated further by

$$
\left|T_{R}^{m}-T_{R}^{\bar{m}}\right| \leqslant \text { constant } \times\left|\left(R_{2}^{m}-R_{2}^{\bar{m}}\right)\left(T_{R}^{m} ; \bar{T}, \bar{R}\right)\right| .
$$

In the third case, estimation of $B^{n+1}-B^{n}$ in a similar manner will lead to expressions of the form

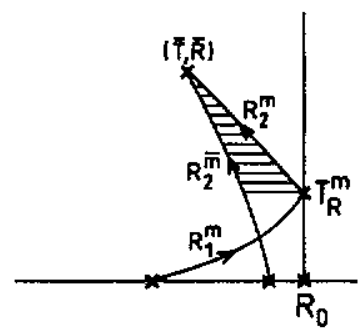

$$
\begin{aligned}
& \text { constant } \times T_{m}^{R} \\
& \text { constant } \times\left(R_{0}-R_{1}^{m}\left(0 ; T_{R}^{m}, R_{0}\right)\right) \\
& \text { constant } \times\left(R_{0}-R_{2}^{\bar{m}}(0 ; \bar{T}, \bar{R})\right)
\end{aligned}
$$

which can all be estimated by $C\left|\left(R_{2}^{m}-R_{2}^{\bar{m}}\right)\left(T_{R}^{m} ; \bar{T}, \bar{R}\right)\right|$ with a suitably chosen constant $C$.

For all these estimates it is crucial that $0<\lambda_{1}<a<\lambda_{2}$, so that the absolute values of the slopes of $R_{1}^{n}$ and $R_{2}^{n}$ are bounded below and above by positive constants. For this reason we had to assume the speed of sound remained positive at the surface of the body.

Next we have to estimate $\left|R_{1,2}^{n}-R_{1.2}^{n-1}\right|$ in terms of $\left|E^{n}-E^{n-1}\right|$. This is done by means of the equation

$$
\left(R_{1.2}^{n}-R_{1,2}^{n-1}\right)(T ; \bar{T}, \bar{R})= \pm \int_{\bar{T}}^{T}\left\{a\left[U^{n}\left(t, R_{1,2}^{n}\right)\right]-a\left[U^{n-1}\left(t, R_{1,2}^{n-1}\right]\right\} \mathrm{d} t\right.
$$

and with help of the Gronwall lemma. (Note that (3.21) is defined only for $T \in J^{n} \cap J^{n-1}$, where $J^{n}$ is the $T$ interval on which $R_{2}^{n}(T ; \bar{T}, \bar{R})$ is defined.) 
Finally, we set

$$
\begin{gathered}
\Delta_{f}^{n}:=\sup _{(T, R) \in \tau}\left\{\sum_{k=1}^{p}\left|\left(D_{k}^{n}-D_{k}^{n-1}\right)(T, R)\right|+\left|\left(A^{n}-A^{n-1}\right)(T, R)\right|\right. \\
\left.+\varepsilon\left|\left(B^{n}-B^{n-1}\right)(T, R)\right|\right\} \quad \varepsilon>0
\end{gathered}
$$

and obtain

$$
\Delta_{f}^{n+1} \leqslant d \Delta_{\varepsilon}^{n}
$$

with a constant $d$ which can be chosen to satisfy $d<1$, provided $T_{0}$ and $\varepsilon$ are chosen sufficiently small. In this case $E^{n}$ converges absolutely and uniformly on $\mathcal{T}$ to a continuous solution $E$ of the integral equations (3.14), (3.15) and (3.17). These equations imply that the characteristic derivatives of the limit functions $E_{j}$ also exist and are continuous, and that (3.11) holds. However, this does not mean that these functions are $C^{\prime}$, let alone $C^{k}$. To prove that they are, we use the fact that, according to the inequalities $(3.18)$, all the functions $E_{j}^{n}$ and their derivatives up to order $k$ satisfy, uniformly on $T$, the same Lipschitz condition (compare [6, ch V, sections 6 and 7]). They form, therefore, a uniformly equicontinuous set. Thus, any sequence $D^{l} E_{j}^{n} \quad(l, j$ fixed) of derivatives contains, according to Ascoli's theorem, a uniformly convergent subsequence, whose limit equals $D^{l} E_{j}$. Hence, the $E_{j}$ are $C^{k}$ functions, as claimed. Uniqueness of the solution can be established by means of an energy estimate. This finishes the proof.

\section{Acknowledgments}

We are grateful to B G Schmidt for drawing attention to the problem treated in the paper and for useful discussions in the course of this work.

\section{References}

[1] Rendall A D and Schmidt B G 1991 Cluss. Quantum Grav. 8985

[2] Bondi H 1947 Mon. Not. R. Astron. Soc. 107410

[3] Rendall A D 1991 J. Math. Phys. 331047

[4] Makino $T$ T 1986 On a local existence theorem for the evolution equation of gaseous stars Patterns and Waves ed T Nishida et al (Amsterdam: North-Holland)

[5] Kind S 1992 Anfangs- Randwertproblem für die Einsteingleichungen zur Beschreibung von Flüssigkeitskugeln und deren linearisierte Störungen PhD Thesis University of Munich

[6] Courant R and Hilbert D 1967 Methods of Mathematical Physics vol 2, Partial Differential Equations (New York: Interscience) ch $\mathrm{V}$, section 7

[7] Garabedian P R 1964 Partial Differential Equations (New York: Wiley) section 4.3

[8] Misner C W and Sharp D H 1964 Phys. Rev. B 136571

[9] Burnett G A 1991 Phys. Rev. D 431143

[10] Buchdahl H A 1959 Phys. Rev. 1161027

[11] Hernandez W C Ir and Misner C W 1966 Astrophys. J. 143452 